\title{
On the Dynamics of a Nonautonomous Predator-Prey Model with Hassell-Varley Type Functional Response
}

\author{
Yan Zhang, ${ }^{1}$ Shujing Gao, ${ }^{1}$ and Kuangang Fan ${ }^{2}$ \\ ${ }^{1}$ Key Laboratory of Jiangxi Province for Numerical Simulation and Emulation Techniques, Gannan Normal University, \\ Ganzhou 341000, China \\ ${ }^{2}$ School of Mechanical and Electrical Engineering, Jiangxi University of Science and Technology, Ganzhou 341000, China
}

Correspondence should be addressed to Yan Zhang; zhyan8401@163.com

Received 27 March 2014; Accepted 21 August 2014; Published 27 October 2014

Academic Editor: Alexander Domoshnitsky

Copyright (C) 2014 Yan Zhang et al. This is an open access article distributed under the Creative Commons Attribution License, which permits unrestricted use, distribution, and reproduction in any medium, provided the original work is properly cited.

\begin{abstract}
The dynamic behaviors of a nonautonomous system for migratory birds with Hassell-Varley type functional response and the saturation incidence rate are studied. Under quite weak assumptions, some sufficient conditions are obtained for the permanence and extinction of the disease. Moreover, the global attractivity of the model is discussed by constructing a Lyapunov function. Numerical simulations which support our theoretical analysis are also given.
\end{abstract}

\section{Introduction}

In the natural world, no species can survive alone. While species spread the disease, compete with the other species for space or food, or are predated by other species, predator-prey relationship can be important in regulating the number of preys and predators. And the dynamic relationship between predators and their preys has long been and will continue to be one of the dominant themes in both ecology and mathematical ecology due to its universal existence and importance [1]. Since the pioneering work of Hadeler and Freedman (1989) of describing a predator-prey model, where the prey is infected by a parasite and in turn infects the predator with the parasite [2], more and more mathematical models for predator-prey behavior are carried out in the following decades; see [3-7] and the references cited therein.

Migratory birds play an important role in the outburst of a new disease and the reintroduction of a disease to a place that was totally washed away from that place on various cases of infectious diseases [8]. For example, the epidemic of Eastern Equine Encephalomyelitis (EEE) which broke in Jamaica in 1962 was suspected to result from transportation of the virus by birds from the continental United States $[9,10]$. As another example, the West Nile Virus was introduced to the Middle East by migrating white storks. Therefore, to control and eradicate infectious diseases spread by the migratory birds has been the key issue in the world as well as in the study of mathematical epidemiology [11].

When investigating biological phenomena, there are many factors which affect dynamical properties of biological and mathematical models. One of the familiar nonlinear factors is functional response. There are many significant functional responses in order to model various different situations. As to predator-prey model, the phenomenon that predators have to share or compete for food is common. Therefore, most of the functional responses, which are assumed to depend on the prey numbers only in most models, are not realistic in the real situation and the predators functional response (i.e., the rate of prey consumption by an average predator) is one of the significant elements which have influence on the relationship between predator and prey $[12$, 13]. The three classical predator-dependent functions are Crowley-Martin type [14], Beddington-DeAngelis type by Beddington [15] and DeAngelis et al. [16], and Hassell-Varley type [17]. A general predator-prey model with Hassell-Varley type functional response may take the following form:

$$
\frac{d x}{d t}=r x\left(1-\frac{x}{K}\right)-\frac{c x y}{m y^{\gamma}+x}
$$




$$
\begin{gathered}
\frac{d y}{d t}=y\left(-d+\frac{f x}{m y^{\gamma}+x}\right), \quad \gamma \in(0,1), \\
x(0)>0, \quad y(0)>0,
\end{gathered}
$$

where $\gamma$ is called Hassell-Varley constant. In a typical predator-prey interaction, where predators do not form groups, one can assume that $\gamma=1$, producing the so-called ratiodependent predator-prey dynamics. For terrestrial predators that form a fixed number of tight groups, it is often reasonable to assume that $\gamma=1 / 2$. For aquatic predators that form a fixed number of tights groups, $\gamma=1 / 3$ may be more appropriate [18]. There are a lot of excellent works on predator-prey models with Hassell-Varley type functional response; for example, see [19-22] and the references therein.

Motivated by these factors, a new nonautonomous predator-prey model with Hassell-Varley type functional response and the saturation incidence rate is proposed to give a more appropriate result and better understanding of the role of migratory birds in pathophoresis. Moreover, under quite weak assumptions, sufficient conditions for the permanence and extinction of the disease are established. In addition, the existence of globally attractive periodic solutions of the system is proposed by discussion and numerical simulation.

The rest of the paper is structured as follows. In the next section, we will introduce the new model. In Section 3, some useful lemmas for one-dimensional nonautonomous equation are proposed. And we establish the sufficient conditions on the permanence and extinction of the disease. Also, by constructing a Lyapunov function, we obtain the global attractivity of the model. Moreover, as applications of the main results, some corollaries are introduced. Particularly, the periodic model is discussed. In Section 4, numerical simulations that verify our qualitative results and a discussion which is about the new model (2) are given. The paper ends with a conclusion.

\section{The Basic Mathematical Model}

In this paper, we propose a predator-prey system, where the predator population $P$ is assumed to be present in the system and the prey population $N=S+I$ migrates into the system. Before we introduce the model, we would like to present a brief sketch of the construction of the model. This may indicate the biological relevance of it.

In the diseases like WNV and avian influenza, it was found that direct transmission in the bird-to-bird transmission of diseases is possible and birds get recovered from the disease, but the duration and variability of immunity among the WNV survivor are essentially unknown [5]; thus, it is reasonable to assume that all the recruitment in the bird population is in the susceptible class $S(t)$ and the infective prey population $I(t)$ is generated through infective of susceptible prey $S(t)$. Also, as time passes, some of the prey population is recovered from the disease at a rate of $f(t)$ and goes to the susceptible class. Furthermore, $\beta(t) S I /(1+\alpha(t) S)$ is the saturation incidence, where $\beta(t)>0$ and $\alpha(t)>0$ measure the force of infective (contact rate) and the force of the inhibition effect at time $t$, respectively.

In the absence of the prey, it is assumed that there exists some alternative food source for the growth of the predator population. The predators eat the susceptible and infected prey with Hassell-Varley type functional response. The growth rate of the predator population is assumed to be $r(t)$ at time $t$. As after the predation of the infective prey, either the infected predators die immediately and thus are removed from the system, or they are dead-end host of the disease like mammals (such as cats) in the case of WNV and in the transmission of many diseases from the migratory birds to their predators, such as highly pathogenic avian influenza (HPAI) virus $(\mathrm{H} 5 \mathrm{~N} 1)[23,24]$. Then we assume that the disease is only spread among the prey population and the disease is not genetically inherited and also the predator becomes infected but the infection does not spread in the predator population.

The above considerations motivate us to introduce the nonautonomous model for the study of the migrating birds under the framework of the following set of nonlinear ordinary differential equations:

$$
\begin{aligned}
\dot{S}(t)= & \Lambda(t)-\frac{\beta(t) S(t) I(t)}{1+\alpha(t) S(t)}-d(t) S(t) \\
& +f(t) I(t)-\frac{c_{1}(t) S(t) P(t)}{g(t) P^{\gamma}(t)+S(t)+I(t)}, \\
\dot{I}(t)= & \frac{\beta(t) S(t) I(t)}{1+\alpha(t) S(t)}-e(t) I(t)-f(t) I(t) \\
& -\frac{c_{2}(t) I(t) P(t)}{g(t) P^{\gamma}(t)+S(t)+I(t)}, \quad \gamma \in(0,1), \\
\dot{P}(t)= & r(t) P(t)\left[1-\frac{P(t)}{K(t)}\right]+\frac{c_{3}(t) S(t) P(t)}{g(t) P^{\gamma}(t)+S(t)+I(t)} \\
& -\frac{c_{4}(t) I(t) P(t)}{g(t) P^{\gamma}(t)+S(t)+I(t)},
\end{aligned}
$$

where $\Lambda(t)$ denotes the instantaneous recruitment rate of the prey population at time $t . d(t)$ denotes the natural death rate of the susceptible prey population and $e(t)$ is the death rate of the infective prey population, which includes the natural death rate and the death rate from the disease, at time $t$. Obviously, $e(t) \geq d(t)$ for all $t \geq 0 . g(t)$ is half-saturation coefficient at time $t$ and $\gamma$ is called a Hassell-Varley constant. And, $c_{1}(t)$ (resp., $\left.c_{2}(t)\right)$ is the maximum value of the per capita rate of $S$ (resp., $I$ ) due to $P$ at time $t$ and $c_{3}(t), c_{4}(t)$ have the similar meaning to $c_{1}(t), c_{2}(t)$. The predators eat both healthy and infected preys at different rates, since the healthy prey more likely escapes from an attack, thus $c_{1}(t) \leq c_{2}(t)$.

The initial conditions are

$$
S(0) \geq 0, \quad I(0) \geq 0, \quad P(0) \geq 0 .
$$

Obviously, the set $\Omega=\left\{(S, I, P) \in R^{3}: S>0, I>0, P>0\right\}$ is a positively invariant set of system (2). 
In this paper, let $C$ denote all continuous functions on the real line. Given $f \in C$, we denote

$$
f^{M}=\sup _{t \geq 0} f(t), \quad f^{m}=\inf _{t \geq 0} f(t) .
$$

If $f$ is $\omega$-periodic, then the average value of $f$ on a time interval $[0, \omega]$ can be defined as

$$
\bar{f}=\frac{1}{\omega} \int_{0}^{\omega} f(t) d t .
$$

\section{Main Results}

In this section, we consider the permanence and extinction of the infective prey. Moreover, by constructing a Lyapunov function and using the comparison theorem, the global attractivity of the model (2) is discussed under two cases; that is, the mutual interference constant $\gamma$ is a rational number and a real-valued number in interval $(0,1)$, respectively. tions:

Firstly, for system (2), we make the following assump-

(H1) functions $\Lambda(t), \beta(t), \alpha(t), d(t), f(t), e(t), r(t), K(t)$, $g(t)$, and $c_{i}(t)(i=1,2,3,4)$ are all nonnegative, continuous, and bounded on $R_{+}=[0,+\infty)$;

$(\mathrm{H} 2)$ there exist positive constants $\omega_{i}>0(i=1,2,3,4)$ such that

$$
\begin{gathered}
\liminf _{t \rightarrow+\infty} \int_{t}^{t+\omega_{1}} \Lambda(\theta) d \theta>0, \quad \liminf _{t \rightarrow+\infty} \int_{t}^{t+\omega_{2}} d(\theta) d \theta>0, \\
\liminf _{t \rightarrow+\infty} \int_{t}^{t+\omega_{3}}\left[r(\theta)-c_{4}(\theta)\right] d \theta>0, \\
\liminf _{t \rightarrow+\infty} \int_{t}^{t+\omega_{4}} \frac{r(\theta)}{K(\theta)} d \theta>0 .
\end{gathered}
$$

For the permanence of the system (2), we have the following theorems.

Theorem 1. Suppose that assumptions (H1) and (H2) hold and there is a constant $w_{5}>0$ such that

$$
\liminf _{t \rightarrow+\infty} \int_{t}^{t+\omega_{5}}\left[\Lambda(\theta)-\left(c_{1}(\theta)+c_{2}(\theta)\right) M_{2}\right] d \theta>0,
$$

where the constant $M_{2}=\left[\left(r+c_{3}\right) K / r\right]^{M}$ is the upper bound of the prey population. Then the prey population $x(t)=S(t)+I(t)$ and the predator population $P(t)$ are permanent.

Proof. Let $(S(t), I(t), P(t))$ be any positive solution of system (2) with initial conditions (3). From the first and second equation of (2), we have

$$
\begin{aligned}
\dot{S}(t)+\dot{I}(t)= & \Lambda(t)-d(t) S(t)-e(t) I(t) \\
& -\frac{\left(c_{1}(t) S(t) P(t)+c_{2}(t) P(t) I(t)\right)}{g(t) P^{\gamma}(t)+S(t)+I(t)} \\
\leq & \Lambda(t)-d(t)(S(t)+I(t)) \quad \forall t \geq 0 .
\end{aligned}
$$

By Lemma 2.1 (see [25]) and the comparison theorem in differential equations, there exist constants $M_{1}>1$ and $T_{1}>$ 0 , such that

$$
S(t)+I(t) \leq M_{1}, \quad \forall t \geq T_{1} .
$$

From the third equation of system (2), we further have

$$
\begin{aligned}
\dot{P}(t)= & r(t) P(t)\left[1-\frac{P(t)}{K(t)}\right] \\
& +\frac{\left(c_{3}(t) S(t) P(t)-c_{4}(t) I(t) P(t)\right)}{g(t) P^{\gamma}(t)+S(t)+I(t)} \\
\leq & P(t)\left[r(t)+c_{3}(t)-\frac{r(t)}{K(t)} P(t)\right]
\end{aligned}
$$

for all $t \geq T_{1}$. By assumption (H2), conclusion (a) of Lemma 1 (see [26]), and the comparison theorem, there exist constants $M_{2}>1$ and $T_{2}\left(\geq T_{1}\right)$, such that

$$
P(t) \leq M_{2}, \quad \forall t \geq T_{2}
$$

Therefore, all solutions $(S, I, P)$ of system (2) with initial conditions (3) are ultimately bounded.

On the other hand, from the first and second equations of (2), we get

$$
\begin{array}{r}
\dot{S}(t)+\dot{I}(t) \geq \Lambda(t)-\left(c_{1}(t)+c_{2}(t)\right) M_{2}-e(t)(S(t)+I(t)), \\
\forall t \geq T_{2} .
\end{array}
$$

By Lemma 2.1 in [25] and the comparison theorem, there are constants $0<m_{1}<1$ and $T_{3}>T_{2}$, such that

$$
S(t)+I(t) \geq m_{1}, \quad \forall t \geq T_{3} .
$$

Further, from the third equation of system (2),

$$
\dot{P}(t) \geq P(t)\left[r(t)-c_{4}(t)-\frac{r(t)}{K(t)} P(t)\right] .
$$

According to the comparison theorem, condition (7), and conclusion (a) of Lemma 1 (see [26]), there exist constants $0<m_{2}<1$ and $T_{4}>T_{3}$ such that

$$
P(t) \geq m_{2}, \quad \forall t \geq T_{4}
$$

Therefore, from (9)-(15), we obtain that

$$
\begin{gathered}
m_{1} \leq \liminf _{t \rightarrow+\infty}(S(t)+I(t)) \leq \limsup _{t \rightarrow+\infty}(S(t)+I(t)) \leq M_{1}, \\
m_{2} \leq \liminf _{t \rightarrow+\infty} P(t) \leq \limsup _{t \rightarrow+\infty} P(t) \leq M_{2} .
\end{gathered}
$$

The proof is completed. 
Remark 2. Suppose that assumptions (H1) and (H2) hold for system (2), and $d^{m}>0,(r / K)^{m}>0$, then we can choose the constants given in the above theorem as follows:

$$
\begin{gathered}
M_{1}=\left(\frac{\Lambda}{d}\right)^{M}, \quad M_{2}=\left(\frac{K r+K c_{3}}{r}\right)^{M}, \\
m_{1}=\left(\frac{\Lambda-\left(c_{1}+c_{2}\right) M_{2}}{e}\right)^{m}, \quad m_{2}=\left(\frac{K r-K c_{4}}{r}\right)^{m} .
\end{gathered}
$$

Let $S_{0}(t)$ be some fixed solution of system

$$
\dot{S}(t)=\Lambda(t)-c_{1}(t) M_{0}-d(t) S,
$$

and $y_{0}(t)$ is a fixed solution of the following nonautonomous Logistic equation:

$$
\dot{y}(t)=y\left(r(t)+c_{3}(t)-\frac{r(t)}{K(t)} y\right),
$$

where $M_{0}=M_{1}+M_{2}$. Then we have the following theorem.

Theorem 3. Suppose that assumptions (H1) and (H2) hold. If there is a constant $\lambda>0$, such that

$$
\begin{gathered}
\liminf _{t \rightarrow+\infty} \frac{1}{\lambda} \int_{t}^{t+\lambda}\left(\frac{\beta(\theta) S_{0}(\theta)}{1+\alpha(\theta) S_{0}(\theta)}-e(\theta)-f(\theta)\right. \\
\left.-\frac{c_{2}(\theta)}{g(\theta)} y_{0}(\theta)\right) d \theta>0,
\end{gathered}
$$

then the infective prey I is permanent.

Proof. Let $(S(t), I(t), P(t))$ be any solution of system (2). From (9)-(20), we can choose sufficiently small $\varepsilon_{1}>0, \varepsilon_{2}>0$; then there exists $T_{1}>0$ such that

$$
\begin{gathered}
\int_{t}^{t+\lambda}\left(\frac{\beta(\theta)\left(S_{0}(\theta)-\varepsilon_{1}\right)}{1+\alpha(\theta)\left(S_{0}(\theta)-\varepsilon_{1}\right)}-e(\theta)-f(\theta)\right. \\
\left.-\frac{c_{2}(\theta)}{g(\theta)}\left(y_{0}(\theta)+\varepsilon_{1}\right)^{1-\gamma}\right) d \theta>\varepsilon_{2}, \\
S(t) \leq M_{0}, \quad I(t) \leq M_{0}, \quad P(t) \leq M_{0}
\end{gathered}
$$

for all $t \geq T_{1}$.

Firstly, we will prove that there exists a positive constant $\epsilon>0$ such that

$$
\limsup _{t \rightarrow \infty} I(t)>\epsilon
$$

Construct an auxiliary equation

$$
\dot{x}(t)=\Lambda(t)-d(t) x(t)-c_{1}(t) M_{0}-\beta(t) M_{0} \alpha_{0} .
$$

By Lemma 4 (see [27]), for the given constants $\varepsilon_{1}>0$ and $M_{0}>0$, there exist positive constants $\delta_{1}=\delta_{1}\left(\varepsilon_{1}\right)>0, B_{1}=$ $B_{1}\left(\varepsilon_{1}, M_{0}\right)>0$, such that for any $t_{0} \in R_{+}$and $x_{0} \in\left[0, M_{0}\right]$, when $\beta(t) M_{0} \alpha_{0}<\delta_{1}$ for all $t \geq t_{0}$, we have

$$
\left|x\left(t, t_{0}, x_{0}\right)-S_{0}(t)\right|<\varepsilon_{1} \quad \forall t \geq t_{0}+B_{1},
$$

where $x\left(t, t_{0}, x_{0}\right)$ is the solution of (24) with initial value $x\left(t_{0}\right)=x_{0}$.

Choose a constant $\alpha_{0}=(1 / 2)\left(\delta_{1} /\left(\beta^{M} M_{0}+1\right)\right)$; we suppose that (23) is not true; then there exists a $Z \in R_{3}^{+}$ such that, for the positive solution $(S(t), I(t), P(t))$ of (2) with initial condition $(S(0), I(0), P(0))=Z$, we have

$$
\limsup _{t \rightarrow \infty} I(t)<\alpha_{0} .
$$

So there is a constant $T_{2}\left(>T_{1}\right)$ such that

$$
I(t)<\alpha_{0}
$$

for all $t \geq T_{2}$. Hence, from the first equation of system (2), we have

$$
\dot{S}(t) \geq \Lambda(t)-c_{1}(t) M_{0}-d(t) S(t)-\beta(t) M_{0} \alpha_{0} .
$$

Let $u(t)$ be the solution of (24) with the condition $x\left(T_{2}\right)=$ $S\left(T_{2}\right)$. In view of comparison theorem, we obtain

$$
S(t) \geq u(t), \quad \forall t \geq T_{2} .
$$

Therefore, from (25), we get

$$
S(t) \geq S_{0}(t)-\varepsilon_{1}, \quad \forall t \geq T_{2}+B_{1} .
$$

From the third equation of system (2), we have

$$
\dot{P}(t) \leq P(t)\left(r(t)-\frac{r(t)}{K(t)} P(t)+c_{3}(t)\right)
$$

for the given constants $\varepsilon_{1}>0$, by comparison theorem, we obtain

$$
P(t) \leq y_{0}(t)+\varepsilon_{1}, \quad \forall t \geq T_{2} .
$$

Therefore, from the second equation of system (2), we further have

$$
\begin{array}{r}
\dot{I}(t) \geq I(t)\left[\frac{\beta(t)\left(S_{0}(t)-\varepsilon_{1}\right)}{1+\alpha(t)\left(S_{0}(t)-\varepsilon_{1}\right)}-(e(t)+f(t))\right. \\
\left.-\frac{c_{2}(t)}{g(t)}\left(y_{0}(t)+\varepsilon_{1}\right)^{1-\gamma}\right], \quad \forall t \geq T^{*},
\end{array}
$$

where $T^{*}=T_{2}+B_{1}$, so

$$
\begin{aligned}
I(t) \geq I\left(T^{*}\right) \exp \int_{T^{*}}^{t} & {\left[\frac{\beta(t)\left(S_{0}(t)-\varepsilon_{1}\right)}{1+\alpha(t)\left(S_{0}(t)-\varepsilon_{1}\right)}-(e(t)+f(t))\right.} \\
& \left.-\frac{c_{2}(t)}{g(t)}\left(y_{0}(t)+\varepsilon_{1}\right)^{1-\gamma}\right] d t .
\end{aligned}
$$

Therefore (21) implies that $I(t) \rightarrow+\infty$, as $t \rightarrow+\infty$. This is a contradiction. Hence, (23) is true.

Thus, for any $t_{0} \geq 0$ we claim that it is impossible that $I(t) \leq \alpha_{0}$, for all $t \geq t_{0}$. From this claim, we will only have to discuss the following possibilities. 
(i) There exists $T \geq T^{*}$, such that $I(t) \geq \alpha_{0}$ for all $t \geq T$.

(ii) $I(t)$ oscillates about $\alpha_{0}$ for all large $t$.

Obviously, we only need to consider case (ii). In the following, we will prove $I(t) \geq \alpha_{0} \exp \left(-\left(h_{1} H+h_{2} \lambda\right)\right) \triangleq m$ for sufficiently large $t$, where

$$
\begin{gathered}
h_{1}=\sup _{t \geq 0}\left[e(t)+f(t)+\frac{c_{2}(t)}{g(t)} M_{0}^{1-\gamma}\right], \\
h_{2}=\sup _{t \geq 0}\left[\frac{\beta(t) S_{0}(t)}{1+\alpha(t) S_{0}(t)}+e(t)+f(t)\right. \\
\left.+\frac{c_{2}(t)}{g(t)}\left(y_{0}(t)+\varepsilon_{1}\right)^{1-\gamma}\right], \\
H=\max \left\{B_{1}, B_{2}\right\} .
\end{gathered}
$$

Let $t_{1}, t_{2}$ be sufficiently large times satisfying

$$
I\left(t_{1}\right)=I\left(t_{2}\right)=\alpha_{0} ; \quad I(t)<\alpha_{0}, \quad \forall t \in\left(t_{1}, t_{2}\right) .
$$

If $t_{2}-t_{1} \leq H$, then from the second equation of system (2)

$$
\begin{aligned}
I(t)=I\left(t_{1}\right) \exp \int_{t_{1}}^{t}\left(\frac{\beta(\theta) S(\theta)}{1+\alpha(\theta) S(\theta)}-e(\theta)-f(\theta)\right. \\
\left.\quad-\frac{c_{2}(\theta) P(\theta)}{g(t) P^{\gamma}+S+I}\right) d \theta \\
\geq I\left(t_{1}\right) \exp \int_{t_{1}}^{t}\left(-e(\theta)-f(\theta)-\frac{c_{2}(\theta)}{g(\theta)} M_{0}^{1-\gamma}\right) d \theta \\
\geq \alpha_{0} \exp \left(-h_{1} H\right), \quad \forall t \in\left[t_{1}, t_{2}\right] .
\end{aligned}
$$

If $t_{2}-t_{1}>H$, being similar to the proof in (30), (32), we know that

$$
S(t) \geq S_{0}(t)-\varepsilon_{1}, \quad P(t) \leq y_{0}(t)+\varepsilon_{1} \quad \forall t \in\left[t_{1}+H, t_{2}\right] .
$$

For any $t \in\left[t_{1}, t_{2}\right]$, if $t \leq t_{1}+H$, from the above discussion, we obtain that

$$
I(t) \geq \alpha_{0} \exp \left(-h_{1} H\right)
$$

If $t \geq t_{1}+H$, letting $q \geq 0$ be a nonnegative integer such that $t \in\left[t_{1}+H+q \lambda, t_{1}+H+(q+1) \lambda\right)$, then from (21), (37), and (38) we have

$$
\begin{gathered}
I(t)=I\left(t_{1}+H\right) \exp \int_{t_{1}+H}^{t}\left(\frac{\beta(\theta) S(\theta)}{1+\alpha(\theta) S(\theta)}-e(\theta)-f(\theta)\right. \\
\left.-\frac{c_{2}(\theta) P(\theta)}{g(t) P^{\gamma}+S+I}\right) d \theta
\end{gathered}
$$

$$
\begin{aligned}
& \geq \alpha_{0} \exp \left(-h_{1} H\right) \\
& \quad \times \exp \int_{t_{1}+H}^{t}\left(\frac{\beta(\theta)\left(S_{0}(\theta)-\varepsilon_{1}\right)}{1+\alpha(\theta)\left(S_{0}(\theta)-\varepsilon_{1}\right)}-e(\theta)\right. \\
& \left.\quad-f(\theta)-\frac{c_{2}(\theta)}{g(\theta)}\left(y_{0}(\theta)+\varepsilon_{1}\right)^{1-\gamma}\right) d \theta \\
& =\alpha_{0} \exp \left(-h_{1} H\right) \\
& \quad \times \exp \left[\int_{t_{1}+H}^{t_{1}+H+q \lambda}+\int_{t_{1}+H+q \lambda}^{t}\right] \\
& \quad \times\left(\frac{\beta(\theta)\left(S_{0}(\theta)-\varepsilon_{1}\right)}{1+\alpha(\theta)\left(S_{0}(\theta)-\varepsilon_{1}\right)}-e(\theta)-f(\theta)\right. \\
& \left.\quad-\frac{c_{2}(\theta)}{g(\theta)}\left(y_{0}(\theta)+\varepsilon_{1}\right)^{1-\gamma}\right) d \theta \\
& \left.\geq \alpha_{0} \exp \left(-h_{1} H\right) \quad-\frac{c_{2}(\theta)}{g(\theta)}\left(y_{0}(\theta)+\varepsilon_{1}\right)^{1-\gamma}\right) d \theta \\
& \quad \times \exp \int_{t_{1}+H+q \lambda}^{t}\left(\frac{\beta(\theta)\left(S_{0}(\theta)-\varepsilon_{1}\right)}{1+\alpha(\theta)\left(S_{0}(\theta)-\varepsilon_{1}\right)}-e(\theta)-f(\theta)\right. \\
& \geq \alpha_{0} \exp \left(-\left(h_{1} H+h_{2} \lambda\right)\right) \triangleq m .
\end{aligned}
$$

Therefore, we have

$$
I(t) \geq m \quad \forall t \in\left[t_{1}, t_{2}\right] .
$$

The proof is completed.

Nextly, we will discuss the extinction of infective $I$ and obtain the following result.

Theorem 4. Suppose that assumptions (H1) and (H2) hold. If there are constants $\gamma, \lambda^{*}>0$, such that

$$
\liminf _{t \rightarrow \infty} \int_{t}^{t+\gamma} \frac{\beta(\theta)}{1+\alpha(\theta) \widetilde{S_{0}}(\theta)} d \theta>0
$$

(H4)

$$
\begin{gathered}
\limsup _{t \rightarrow+\infty} \frac{1}{\lambda^{*}} \int_{t}^{t+\lambda^{*}}\left(\frac{\beta(\theta) \widetilde{S_{0}}(\theta)}{1+\alpha(\theta) \widetilde{S_{0}}(\theta)}-e(\theta)-f(\theta)\right. \\
\left.-\frac{c_{2}(\theta) \widetilde{y_{0}}(\theta)}{(g(\theta)+2) M_{0}}\right) d \theta \leq 0
\end{gathered}
$$

where $\widetilde{S_{0}}(t)$ is some fixed solution of system

$$
\dot{S}(t)=\Lambda(t)-d(t) S,
$$


and $\widetilde{y_{0}}(t)$ is a fixed solution of the following nonautonomous Logistic equation:

$$
\dot{y}(t)=y\left(r(t)-c_{4}(t)-\frac{r(t)}{K(t)} y\right),
$$

then the infective prey $I$ is extinct.

Proof. From assumption (H3), we can choose $\eta>0$ small enough and $T_{0}>0$ big enough satisfying

$$
\int_{t}^{t+\gamma} \frac{\beta(\theta)}{1+\alpha(\theta) \widetilde{S_{0}}(\theta)} d \theta \geq \eta, \quad \forall t \geq T_{0} .
$$

For any $0<\sigma<1$, we set $\sigma_{0}=\min \left\{\lambda^{*} \eta \sigma / 2 \gamma,(1 / 2) \eta \sigma\right\}$. If (H4) holds, then there exist $\delta>0$ and $T_{1} \geq T_{0}$ such that

$$
\begin{gathered}
\int_{t}^{t+\lambda^{*}}\left(\frac{\beta(\theta)\left(\widetilde{S_{0}}(\theta)+\delta\right)}{1+\alpha(\theta)\left(\widetilde{S_{0}}(\theta)+\delta\right)}-e(\theta)-f(\theta)\right. \\
\left.-\frac{c_{2}(\theta)\left(\widetilde{y_{0}}(\theta)-\delta\right)}{\left(g(\theta+2) M_{0}\right)}\right) d \theta \leq \sigma_{0}
\end{gathered}
$$

for all $t \geq T_{1}$. Choose an integer $n_{0}$ satisfying $2 \gamma / \lambda^{*} \leq n_{0} \leq$ $\left(2 \gamma / \lambda^{*}\right)+1$. Set $\lambda_{0}=n_{0} \lambda^{*}$; then

$$
\begin{aligned}
& \int_{t}^{t+\lambda_{0}}\left(\frac{\beta(\theta)\left(\widetilde{S_{0}}(\theta)+\delta\right)}{1+\alpha(\theta)\left(\widetilde{S_{0}}(\theta)+\delta\right)}-e(\theta)-f(\theta)\right. \\
& \left.\quad-\frac{c_{2}(\theta)\left(\widetilde{y_{0}}(\theta)-\delta\right)}{\left(g(\theta+2) M_{0}\right)}-\frac{\beta(\theta) \sigma}{1+\alpha(\theta)\left(\widetilde{S_{0}}(\theta)+\delta\right)}\right) d \theta \\
& \leq \int_{t}^{t+n_{0} \lambda^{*}}\left(\frac{\beta(\theta)\left(\widetilde{S_{0}}(\theta)+\delta\right)}{1+\alpha(\theta)\left(\widetilde{S_{0}}(\theta)+\delta\right)}-e(\theta)-f(\theta)\right. \\
& \left.-\int_{t}^{t+2 \gamma} \frac{c_{2}(\theta)\left(\widetilde{y_{0}}(\theta)-\delta\right)}{\left(g(\theta+2) M_{0}\right)}\right) d \theta \\
& \leq n_{0} \sigma_{0}-2 \eta \sigma \leq-\frac{1}{2} \eta \sigma .
\end{aligned}
$$

Differentiating $S+I$ along a solution of system (2), by the comparison theorem and Lemma 2.1 (b) (see [25]), there exists a constant $T_{2} \geq T_{1}$ such that

$$
S(t)+I(t) \leq \widetilde{S_{0}}(t)+\delta, \quad \forall t \geq T_{2}
$$

Further, from the third equation of system (2) and Lemma 1 (b) (see [26]), there is a $T_{3} \geq T_{2}$ such that

$$
P(t) \geq \widetilde{y_{0}}(t)-\delta, \quad \forall t \geq T_{3} .
$$

Let $T=\max \left\{T_{2}, T_{3}\right\}, h=\sup _{t \geq T}\left\{\beta(t)\left(\widetilde{S_{0}}(t)+\delta\right)+e(t)+\right.$ $\left.f(t)+\left(c_{2}(t) / 2 M_{0}\right)\left(\widetilde{y_{0}}(t)+\delta\right)+\beta(t)\right\}$; then, for all $t \geq T$, we have

$$
\begin{aligned}
& \dot{I}(t) \leq I(t)\left[\frac{\beta(t)\left(\widetilde{S_{0}}(t)+\delta-I(t)\right)}{1+\alpha(t)\left(\widetilde{S_{0}}(t)+\delta\right)}-e(t)-f(t)\right. \\
&\left.-\frac{c_{2}(t)}{(g(t)+2) M_{0}}\left(\widetilde{y_{0}}(t)-\delta\right)\right] .
\end{aligned}
$$

If $I(t) \geq \sigma$ for all $t \geq T$, then let $q^{*} \geq 0$ be a nonnegative integer such that $t \in\left[T+q^{*} \lambda_{0}, T+\left(q^{*}+1\right) \lambda_{0}\right)$; integrating (51) from $T$ to $t$, we obtain

$I(t)$

$\leq I(T)$

$$
\begin{aligned}
\times \exp \int_{T}^{t}\left[\frac{\beta(\theta)\left(\widetilde{S_{0}}(\theta)+\delta\right)}{1+\alpha(\theta)\left(\widetilde{S_{0}}(\theta)+\delta\right)}-e(\theta)-f(\theta)\right. \\
\left.\quad-\frac{c_{2}(\theta)\left(\widetilde{y_{0}}(\theta)-\delta\right)}{(g(\theta)+2) M_{0}}-\frac{\beta(\theta) \sigma}{1+\alpha(\theta)\left(\widetilde{S_{0}}(\theta)+\delta\right)}\right] d \theta \\
=I(T) \exp \left[\int_{T}^{T+q^{*} \lambda_{0}}+\int_{T+q^{*} \lambda_{0}}^{t}\right] \\
\times\left\{\frac{\beta(\theta)\left(\widetilde{S_{0}}(\theta)+\delta\right)}{1+\alpha(\theta) \widetilde{S_{0}}(\theta)}-e(\theta)-f(\theta)\right. \\
\left.\quad-\frac{c_{2}(\theta)\left(\widetilde{y_{0}}(\theta)-\delta\right)}{(g(\theta)+2) M_{0}}-\frac{\beta(\theta) \sigma}{1+\alpha(\theta) \widetilde{S_{0}}(\theta)}\right\} d \theta \\
\leq I(T) \exp \left[-\frac{1}{2} \eta \sigma q^{*}\right] \exp \left(\lambda_{0} h\right) .
\end{aligned}
$$

Then it follows that $I(t) \rightarrow 0$ as $t \rightarrow+\infty$. This is a contradiction with $I(t) \geq \sigma$. Hence there must be a $t_{1} \geq T$ such that $I\left(t_{1}\right)<\sigma$.

Finally, we will prove

$$
I(t) \leq \sigma \exp \left(h \lambda_{0}\right)
$$

for all $t \geq t_{1}$. If it is not true, there exists a $t_{2}>t_{1}$ such that $I\left(t_{2}\right)>\sigma \exp \left(h \lambda_{0}\right)$. Hence, there exists a $t_{3} \in\left(t_{1}, t_{2}\right)$ such that $I\left(t_{3}\right)=\sigma$ and $I(t)>\sigma$ for all $t \in\left(t_{3}, t_{2}\right)$. Let $l_{1}$ be a nonnegative integer such that $t_{2} \in\left[t_{3}+l_{1} \lambda_{0}, t_{3}+\left(l_{1}+1\right) \lambda_{0}\right)$; then integrating (51) from $t_{3}$ to $t_{2}$, we obtain

$$
\begin{aligned}
& \sigma \exp \left(h \lambda_{0}\right) \\
& \leq I\left(t_{2}\right) \leq I\left(t_{3}\right) \\
& \quad \times \exp \int_{t_{3}}^{t_{2}}\left[\frac{\beta(t)\left(\widetilde{S_{0}}(t)+\delta\right)}{1+\alpha(t)\left(\widetilde{S_{0}}(t)+\delta\right)}-e(t)-f(t)\right. \\
& \left.\quad-\frac{c_{2}(\theta)\left(\widetilde{y_{0}}(\theta)-\delta\right)}{(g(\theta)+2) M_{0}}-\frac{\beta(t) I(t)}{1+\alpha(t)\left(\widetilde{S_{0}}(t)+\delta\right)}\right] d t
\end{aligned}
$$




$$
\begin{aligned}
& \leq I\left(t_{3}\right) \exp \left[\int_{t_{3}}^{t_{3}+l_{1} \lambda_{0}}+\int_{t_{3}+l_{1} \lambda_{0}}^{t_{2}}\right] \\
& \times\left[\frac{\beta(t)\left(\widetilde{S_{0}}(t)+\delta\right)}{1+\alpha(t)\left(\widetilde{S_{0}}(t)+\delta\right)}-e(t)-f(t)\right. \\
& \left.\quad-\frac{c_{2}(\theta)\left(\widetilde{y_{0}}(\theta)-\delta\right)}{(g(\theta)+2) M_{0}}-\frac{\beta(t) \sigma}{1+\alpha(t)\left(\widetilde{S_{0}}(t)+\delta\right)}\right] d t \\
& \leq \sigma \exp \left(-\frac{1}{2} \eta \sigma l_{1}\right) \exp \left(\lambda_{0} h\right) .
\end{aligned}
$$

This leads to a contradiction. Hence, inequality (53) holds. Furthermore, since $\sigma$ can be arbitrarily small, we conclude that $I(t) \rightarrow 0$, as $t \rightarrow+\infty$. The proof is completed.

In particular, when system (2) degenerates into an $\omega$-periodic system, then assumptions $(\mathrm{H1}),(\mathrm{H} 2)$, and $(\mathrm{H} 3)$ are equivalent to the following forms:

(C1) functions $\Lambda(t), \beta(t), \alpha(t), d(t), f(t), e(t), r(t), K(t)$, $g(t)$, and $c_{i}(t)(i=1,2,3,4)$ are all nonnegative, continuous periodic functions with a period $\omega>0$;

(C2) $\bar{\Lambda}>0, \bar{d}>0, \overline{r-c_{4}}>0, \overline{(r / K)}>0$;

(C3) $\overline{\beta /\left(1+\alpha \widetilde{S_{0}}\right)}>0$.

As consequences of Theorems 3 and 4, we have the following corollaries.

Corollary 5. Suppose that assumptions (C1) and (C2) hold, and

$$
R_{*}=\frac{\overline{\beta S_{0} /\left(1+\alpha S_{0}\right)}}{\left(\bar{e}+\bar{f}+\overline{c_{2} y_{0} / g}\right)}>1 ;
$$

then the infective I of system (2) is permanent.

Corollary 6. Suppose that assumptions (C1), (C2), and (C3) hold, and

$$
R^{*}=\frac{\overline{\beta \widetilde{S}_{0} /\left(1+\alpha \widetilde{S_{0}}\right)}}{\left(\bar{e}+\bar{f}+\overline{c_{2} \widetilde{y_{0}} /(g+2) M_{0}}\right)} \leq 1 ;
$$

then the infective I of system (2) is extinct.

Lastly, we will give the discussion on the global attractivity of model (2) as follows:

Theorem 7. Suppose that assumptions (H1) and (H2) hold and $\gamma \in(0,1)$ is a rational number. If there are constants $\mu_{i}>0(i=1,2,3)$ such that $\liminf _{t \rightarrow \infty} A_{i}(t)>0$ and $\liminf _{t \rightarrow \infty}\left(M_{2}^{1-\gamma} A_{3}(t)+A_{4}(t)\right)>0$, where

$$
\begin{aligned}
& A_{1}(t) \\
& =\mu_{1}\left[d(t)-\left(c_{2}(t)-c_{1}(t)\right) \frac{M_{1} M_{2}^{1-\gamma}}{m_{1}}\right]
\end{aligned}
$$

$$
\begin{aligned}
& -\mu_{2}\left[\beta(t)-\frac{M_{2}^{1-\gamma} c_{2}(t)}{m_{1} g(t)}\right] \\
& -\mu_{3}\left[\frac{c_{3}(t)}{m_{1}}+\frac{\left(c_{3}(t)+c_{4}(t)\right) M_{1}}{m_{1}^{2}}\right],
\end{aligned}
$$

$A_{2}(t)$

$$
\begin{aligned}
= & \mu_{2} \frac{\beta(t)}{\left(1+\alpha(t) M_{1}\right)^{2}} \\
& -\mu_{1}\left[e(t)-d(t)+\left(c_{2}(t)-c_{1}(t)\right)\left(M_{2}^{1-\gamma}+\frac{M_{2}}{m_{1}}\right)\right] \\
& -\mu_{1}\left[c_{2}(t)-c_{1}(t)\right] M_{1}-2 \mu_{3} \frac{\left[c_{3}(t)+c_{4}(t)\right]}{m_{1}}
\end{aligned}
$$

$A_{3}(t)$

$$
\begin{aligned}
= & \mu_{3} \frac{r(t)}{K(t)}-\mu_{1}\left[2 c_{2}(t)+\frac{M_{1}}{m_{1}}\left(c_{2}(t)-c_{1}(t)\right) g(t)\right] \\
& -\mu_{2} \frac{2 c_{2}(t)}{m_{1}},
\end{aligned}
$$

$A_{4}(t)$

$$
\begin{aligned}
= & -\mu_{1}\left[M_{2}^{2-\gamma} c_{1}(t)-\left(c_{2}(t)-c_{1}(t)\right) g(t) \frac{M_{1} M_{2}}{m_{1}^{2}}\right] \\
& -\mu_{2} c_{2}(t) \frac{M_{2}^{1-\gamma}}{m_{1}}-\mu_{3} g(t)\left[\frac{c_{3}(t)}{m_{1}}+\frac{\left(c_{3}(t)+c_{4}(t)\right) M_{1}}{m_{1}^{2}}\right],
\end{aligned}
$$

and $M_{1}, M_{2}$ are the constants obtained in Theorem 1, then model (2) is globally attractive.

Proof. Let $x=S+I$; then system (2) is equivalent to the following system:

$$
\begin{aligned}
\dot{x}(t)= & \Lambda(t)-d(t) x-[e(t)-d(t)] I \\
& -\frac{c_{1}(t) x P}{g(t) P^{\gamma}+x}+\frac{\left[c_{1}(t)-c_{2}(t)\right] P I}{g(t) P^{\gamma}+x},
\end{aligned}
$$

$$
\begin{gathered}
\dot{I}(t)=I\left[\frac{\beta(t)(x-I)}{1+\alpha(t)(x-I)}-e(t)-f(t)-\frac{c_{2}(t) P}{g(t) P^{\gamma}+x}\right], \\
\dot{P}(t)=P\left[r(t)+\frac{c_{3}(t) x}{g(t) P^{\gamma}+x}-\frac{\left(c_{3}(t)+c_{4}(t)\right) I}{g(t) P^{\gamma}+x}-\frac{r(t)}{K(t)} P\right] .
\end{gathered}
$$

Let $\left(x_{1}(t), I_{1}(t), P_{1}(t)\right),\left(x_{2}(t), I_{2}(t), P_{2}(t)\right)$ be any two solutions of system (58). Then, from (9)-(13), we have

$$
\begin{array}{r}
m_{1} \leq x_{k}(t) \leq M_{1}, \quad I_{k}(t) \leq M_{1}, \quad P_{k}(t) \leq M_{2}, \\
\forall t \geq 0, \quad k=1,2 .
\end{array}
$$


Construct a Lyapunov function

$$
\begin{aligned}
V(t)= & \mu_{1}\left|x_{1}(t)-x_{2}(t)\right| \\
& +\mu_{2}\left|\ln I_{1}(t)-\ln I_{2}(t)\right|+\mu_{3}\left|\ln P_{1}(t)-\ln P_{2}(t)\right| .
\end{aligned}
$$

Thus

$$
D^{+}(V(t))
$$$$
=\mu_{1} \operatorname{sgn}\left(x_{1}-x_{2}\right)
$$$$
\times\left\{-d(t)\left(x_{1}-x_{2}\right)-(e(t)-d(t))\left(I_{1}-I_{2}\right)\right.
$$$$
-c_{1}(t)\left(\frac{P_{1} x_{1}}{g(t) P_{1}^{\gamma}+x_{1}}-\frac{P_{2} x_{2}}{g(t) P_{2}^{\gamma}+x_{2}}\right)
$$$$
\left.+\left[c_{1}(t)-c_{2}(t)\right]\left(\frac{P_{1} I_{1}}{g(t) P_{1}^{\gamma}+x_{1}}-\frac{P_{2} I_{2}}{g(t) P_{2}^{\gamma}+x_{2}}\right)\right\}
$$$$
+\mu_{2} \operatorname{sgn}\left(I_{1}-I_{2}\right)
$$$$
\times\left\{\frac{\beta(t)\left(x_{1}-I_{1}\right)}{1+\alpha(t)\left(x_{1}-I_{1}\right)}-\frac{\beta(t)\left(x_{2}-I_{2}\right)}{1+\alpha(t)\left(x_{2}-I_{2}\right)}\right.
$$$$
\left.-c_{2}(t)\left(\frac{P_{1}}{g(t) P_{1}^{\gamma}+x_{1}}-\frac{P_{2}}{g(t) P_{2}^{\gamma}+x_{2}}\right)\right\}
$$$$
+\mu_{3} \operatorname{sgn}\left(P_{1}-P_{2}\right)
$$$$
\times\left\{c_{3}(t)\left(\frac{x_{1}}{g(t) P_{1}^{\gamma}+x_{1}}-\frac{x_{2}}{g(t) P_{2}^{\gamma}+x_{2}}\right)\right.
$$$$
\left.-\left(c_{3}(t)+c_{4}(t)\right)\left(\frac{I_{1}}{g(t) P_{1}^{\gamma}+x_{1}}-\frac{I_{2}}{g(t) P_{2}^{\gamma}+x_{2}}\right)\right\}
$$$$
-\mu_{3} \frac{r(t)}{k(t)}\left(P_{1}-P_{2}\right)
$$$$
\leq \mu_{1}\left\{-d(t)\left|x_{1}-x_{2}\right|+(e(t)-d(t))\left|I_{1}-I_{2}\right|+c_{1}(t) M_{2}^{2-\gamma}\right.
$$$$
\times\left|P_{1}^{\gamma}-P_{2}^{\gamma}\right|+2 c_{1}(t) M_{1}\left|P_{1}-P_{2}\right|
$$$$
+g(t)\left[k_{2}(t)-k_{1}(t)\right] \frac{M_{1} M_{2}}{m_{1}^{2}}\left|P_{1}^{\gamma}-P_{2}^{\gamma}\right|
$$$$
+\left[c_{2}(t)-c_{1}(t)\right] \frac{M_{1}}{m_{1}}\left|I_{1}-I_{2}\right|
$$$$
+\left(c_{2}(t)-c_{1}(t)\right) \frac{M_{2}^{1-\gamma} M_{1}}{m_{1}}\left|x_{1}-x_{2}\right|
$$$$
+\left(c_{2}(t)-c_{1}(t)\right) M_{2}^{1-\gamma}
$$$$
\left.\times\left|I_{1}-I_{2}\right| g(t)\left(c_{2}(t)-c_{1}(t)\right) \frac{M_{1}}{m_{1}}\left|P_{1}-P_{2}\right|\right\}
$$

$$
+\mu_{2}\left\{\beta(t)\left|x_{1}-x_{2}\right|-\frac{\beta(t)}{\left(1+\alpha(t) M_{1}\right)^{2}}\left|I_{1}-I_{2}\right|\right.
$$$$
+2 c_{2}(t) \frac{1}{m_{1}}\left|P_{1}-P_{2}\right|+\frac{M_{2}^{1-\gamma} c_{2}(t)}{m_{1} g(t)}
$$

$$
\left.\times\left|x_{1}-x_{2}\right|+c_{2}(t) \frac{M_{2}^{1-\gamma}}{m_{1}}\left|P_{1}^{\gamma}-P_{2}^{\gamma}\right|\right\}
$$

$$
\begin{aligned}
+\mu_{3}\left\{\frac{c_{3}(t)}{m_{1}}\left|x_{1}-x_{2}\right|+2 \frac{\left(c_{3}(t)+c_{4}(t)\right)}{m_{1}}\left|I_{1}-I_{2}\right|\right. \\
+\frac{\left(c_{3}(t)+c_{4}(t)\right) M_{1}}{m_{1}^{2}}\left|x_{1}-x_{2}\right| \\
+\frac{\left[\left(m_{1}+M_{1}\right) c_{3}(t)+M_{1} c_{4}(t)\right]}{m_{1}^{2}} g(t)\left|P_{1}^{\gamma}-P_{2}^{\gamma}\right| \\
\left.+\frac{r(t)}{k(t)}\left|P_{1}-P_{2}\right|\right\}
\end{aligned}
$$

$$
\begin{aligned}
=\{ & -\mu_{1}\left[d(t)-\left(c_{2}(t)-c_{1}(t)\right) \frac{M_{1} M_{2}^{1-\gamma}}{m_{1}}\right] \\
& \left.+\mu_{2}\left[\beta(t)-\frac{M_{2}^{1-\gamma} c_{2}(t)}{m_{1} g(t)}\right]+\mu_{3} \frac{\left(c_{3}(t)+c_{4}(t)\right) M_{1}}{m_{1}^{2}}\right\}
\end{aligned}
$$

$$
\times\left|x_{1}-x_{2}\right|
$$$$
+\left\{\mu_{1}\left[e(t)-d(t)+\left(c_{2}(t)-c_{1}(t)\right)\left(M_{2}^{1-\gamma}+\frac{M_{2}}{m_{1}}\right)\right]\right.
$$$$
\left.-\mu_{2} \frac{\beta(t)}{\left(1+\alpha(t) M_{1}\right)^{2}}+\mu_{1}\left[c_{2}(t)-c_{1}(t)\right] M_{1}\right\}\left|I_{1}-I_{2}\right|
$$$$
+2 \mu_{3} \frac{\left[c_{3}(t)+c_{4}(t)\right]}{m_{1}}\left|I_{1}-I_{2}\right|+\mu_{3} \frac{c_{3}(t)}{m_{1}}\left|x_{1}-x_{2}\right|
$$

$$
=\left\{-\mu_{3} \frac{r(t)}{K(t)}+\mu_{1}\left[2 c_{2}(t)+\frac{M_{1}}{m_{1}}\left(c_{2}(t)-c_{1}(t)\right) g(t)\right]\right.
$$$$
\left.+\mu_{2} \frac{2 c_{2}(t)}{m_{1}}\right\}\left|P_{1}-P_{2}\right|
$$

$$
=\left\{\mu_{1}\left[M_{2}^{2-\gamma} c_{1}(t)-\left(c_{2}(t)-c_{1}(t)\right) g(t) \frac{M_{1} M_{2}}{m_{1}^{2}}\right]\right.
$$

$$
\left.+\mu_{2} c_{2}(t) \frac{M_{2}^{1-\gamma}}{m_{1}}+\mu_{3} g(t)\left[\frac{c_{3}(t)}{m_{1}}+\frac{\left(c_{3}(t)+c_{4}(t)\right) M_{1}}{m_{1}^{2}}\right]\right\}
$$

$$
\times\left|P_{1}^{\gamma}-P_{2}^{\gamma}\right|
$$

$$
\begin{aligned}
= & -A_{1}(t)\left|x_{1}-x_{2}\right|-A_{2}(t)\left|I_{1}-I_{2}\right| \\
& -A_{3}(t)\left|P_{1}-P_{2}\right|-A_{4}(t)\left|P_{1}^{\gamma}-P_{2}^{\gamma}\right| .
\end{aligned}
$$


Note that $\gamma$ is a rational number, which yields that there exist two mutually prime numbers $p$ and $q$ with $p>q$ and $\gamma=q / p$, such that

$$
\begin{aligned}
P_{1}^{\gamma}(t)-P_{2}^{\gamma}(t) & \\
= & {\left[P_{1}^{1 / p}(t)-P_{2}^{1 / p}(t)\right] \sum_{i=1}^{q} P_{1}^{(q-i) / p}(t) P_{2}^{(i-1) / p}(t), } \\
P_{1}(t)-P_{2}(t) & \\
= & {\left[P_{1}^{1 / p}(t)-P_{2}^{1 / p}(t)\right] \sum_{i=1}^{p} P_{1}^{(p-i) / p}(t) P_{2}^{(i-1) / p}(t) . }
\end{aligned}
$$

Then, we can obtain that

$$
\begin{aligned}
& D^{+}(V(t)) \\
& \leq-A_{1}(t)\left|x_{1}-x_{2}\right|-A_{2}(t)\left|I_{1}-I_{2}\right| \\
& -\left\{A_{3}(t) \sum_{i=1}^{p} P_{1}^{(p-i) / p}(t) P_{2}^{(i-1) / p}(t)\right. \\
& \left.+A_{4}(t) \sum_{i=1}^{q} P_{1}^{(q-i) / p}(t) P_{2}^{(i-1) / p}(t)\right\} \\
& \times\left|P_{1}^{1 / p}(t)-P_{2}^{1 / p}(t)\right| \\
& =-A_{1}(t)\left|x_{1}-x_{2}\right|-A_{2}(t)\left|I_{1}-I_{2}\right| \\
& -A_{3}(t) \sum_{i=q}^{p} P_{1}^{(p-i) / p}(t) P_{2}^{(i-1) / p}(t)\left|P_{1}^{1 / p}(t)-P_{2}^{1 / p}(t)\right| \\
& -\left[A_{3}(t) \sum_{i=1}^{q} P_{1}^{(p-i) / p}(t) P_{2}^{(i-1) / p}(t)\right. \\
& \left.+A_{4}(t) \sum_{i=1}^{q} P_{1}^{(q-i) / p}(t) P_{2}^{(i-1) / p}(t)\right] \\
& \times\left|P_{1}^{1 / p}(t)-P_{2}^{1 / p}(t)\right| \\
& =-A_{1}(t)\left|x_{1}-x_{2}\right|-A_{2}(t)\left|I_{1}-I_{2}\right| \\
& -A_{3}(t) \sum_{i=q}^{p} P_{1}^{(p-i) / p}(t) P_{2}^{(i-1) / p}(t)\left|P_{1}^{1 / p}(t)-P_{2}^{1 / p}(t)\right| \\
& -\left[A_{3}(t) P_{1}^{\gamma-1}(t)+A_{4}(t)\right] \\
& \times \sum_{i=1}^{q} P_{1}^{(q-i) / p}(t) P_{2}^{(i-1) / p}(t)\left|P_{1}^{1 / p}(t)-P_{2}^{1 / p}(t)\right| \\
& \leq-A_{1}(t)\left|x_{1}-x_{2}\right|-A_{2}(t)\left|I_{1}-I_{2}\right| \\
& -A_{3}(t) \sum_{i=q}^{p} P_{1}^{(p-i) / p}(t) P_{2}^{(i-1) / p}(t)\left|P_{1}^{1 / p}(t)-P_{2}^{1 / p}(t)\right| \\
& -\left[A_{3}(t) M_{2}^{\gamma-1}(t)+A_{4}(t)\right] \\
& \times \sum_{i=1}^{q} P_{1}^{(q-i) / p}(t) P_{2}^{(i-1) / p}(t)\left|P_{1}^{1 / p}(t)-P_{2}^{1 / p}(t)\right| .
\end{aligned}
$$

Since $\liminf _{t \rightarrow \infty} A_{i}(t)>0(i=1,2,3)$ and $\liminf _{t \rightarrow \infty}$ $\left(M_{2}^{1-\gamma} A_{3}(t)+A_{4}(t)\right)>0$, there exist constants $\alpha>0$ and $T_{0}>$ 0 such that $A_{i}(t) \geq \alpha(i=1,2,3)$ for all $t \geq T_{0}$. Furthermore, we obtain that

$$
\begin{aligned}
D^{+}(V(t)) \leq-\alpha & \left(\left|x_{1}(t)-x_{2}(t)\right|+\left|I_{1}(t)-I_{2}(t)\right|\right. \\
& \left.+\left|P_{1}(t)-P_{2}(t)\right|+\left|P_{1}^{1 / p}(t)-P_{2}^{1 / p}(t)\right|\right)
\end{aligned}
$$

for all $t \geq T_{0}$. Taking integral on both sides of (64) from $T_{0}$ to $t$, then

$$
\begin{aligned}
V(t)-V\left(T_{0}\right) & \\
\leq-\alpha \int_{T_{0}}^{t}( & \left|x_{1}(s)-x_{2}(s)\right|+\left|I_{1}(s)-I_{2}(s)\right| \\
& \left.\quad+\left|P_{1}(s)-P_{2}(s)\right|+\left|P_{1}^{1 / p}(s)-P_{2}^{1 / p}(s)\right|\right) d s
\end{aligned}
$$

that is,

$$
\begin{gathered}
\alpha \int_{T_{0}}^{t}\left(\left|x_{1}(s)-x_{2}(s)\right|+\left|I_{1}(s)-I_{2}(s)\right|+\left|P_{1}(s)-P_{2}(s)\right|\right. \\
\left.+\left|P_{1}^{1 / p}(s)-P_{2}^{1 / p}(s)\right|\right) d s \leq V\left(T_{0}\right)<+\infty .
\end{gathered}
$$

On the other hand, from (58) and (59), we can get that $(d / d t)$ $\left(x_{1}-x_{2}\right),(d / d t)\left(I_{1}-I_{2}\right)$, and $(d / d t)\left(P_{1}-P_{2}\right)$ are all bounded on $[0, \infty)$. So from $(66)$,

$$
\begin{gathered}
\lim _{t \rightarrow \infty}\left|x_{1}(t)-x_{2}(t)\right|=0, \quad \lim _{t \rightarrow \infty}\left|I_{1}(t)-I_{2}(t)\right|=0, \\
\lim _{t \rightarrow \infty}\left|P_{1}(t)-P_{2}(t)\right|=0 .
\end{gathered}
$$

The proof is completed.

Theorem 8. If we replace the term $M_{2}^{1-\gamma} A_{3}(t)+A_{4}(t)$ in Theorem 7 by $A_{3}(t)+\gamma M_{2}^{\gamma-1} A_{4}(t)$, then the result still holds without the need for the assumption that $\gamma$ is a rational number.

Proof. The mean value theorem yields that there is $\xi \in$ $\left(m_{2}, M_{2}\right)$ such that

$$
\begin{aligned}
\left|P_{1}^{\gamma}(t)-P_{2}^{\gamma}(t)\right| & =\gamma \xi^{\gamma-1}\left|P_{1}(t)-P_{2}(t)\right| \\
& \geq \gamma M_{2}^{\gamma-1}\left|P_{1}(t)-P_{2}(t)\right|,
\end{aligned}
$$

which, together with (61), makes us obtain that

$$
\begin{aligned}
D^{+} V(t) \leq & -A_{1}(t)\left|x_{1}(t)-x_{2}(t)\right|-A_{2}(t)\left|I_{1}(t)-I_{2}(t)\right| \\
& -\left[A_{3}(t)+\gamma M_{2}^{\gamma-1} A_{4}(t)\right]\left|P_{1}(t)-P_{2}(t)\right| .
\end{aligned}
$$

The remainder of the proof is similar to the previous proof of Theorem 7. Here we omit it. 


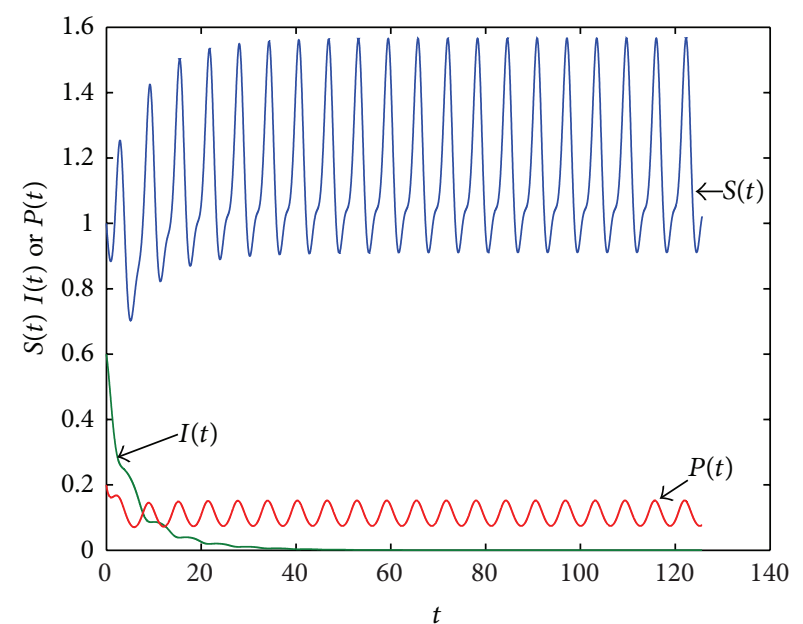

(a)

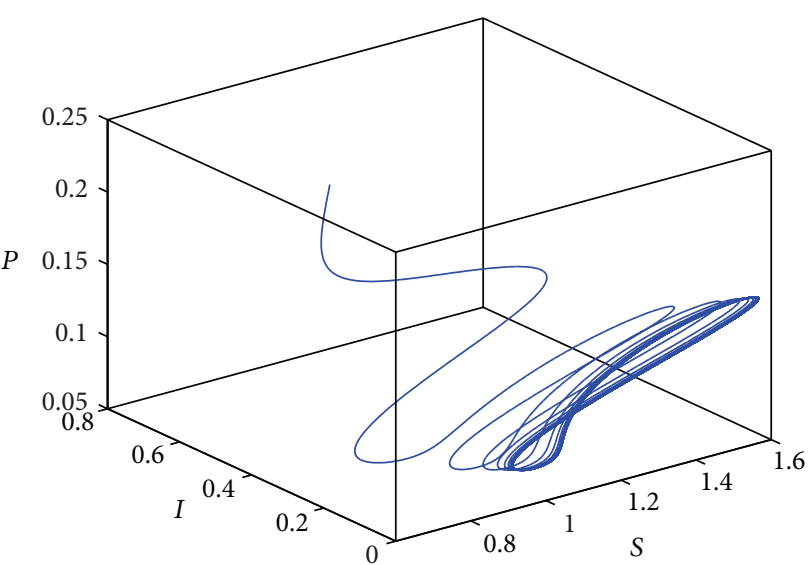

(b)

Figure 1: (a) The movement paths of $S, I$, and $P$ as functions of time $t$. The graph of the trajectory in $(S, I, P)$-Space is shown in (b). $R^{*}=$ $0.9898<1$. The disease is extinct.

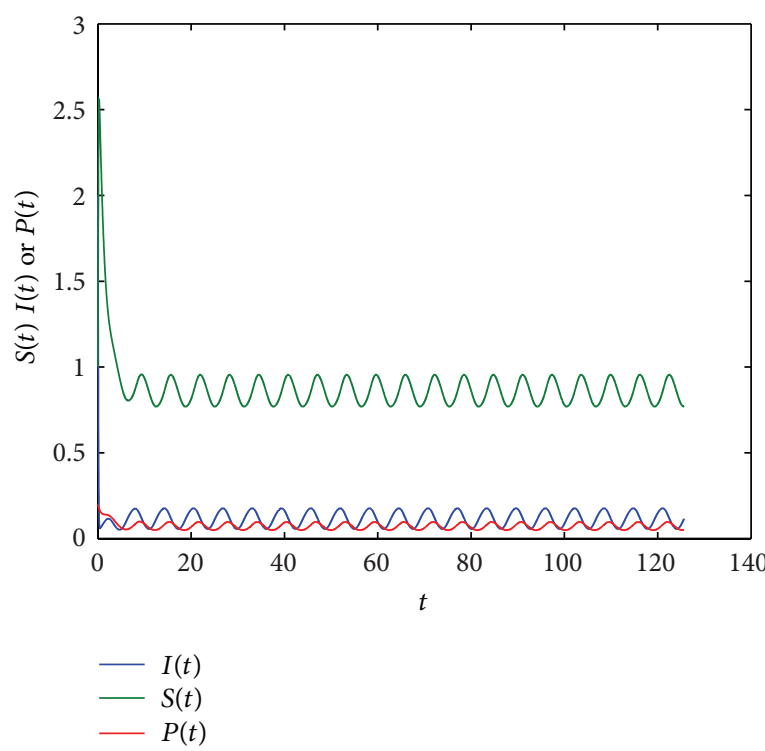

(a)

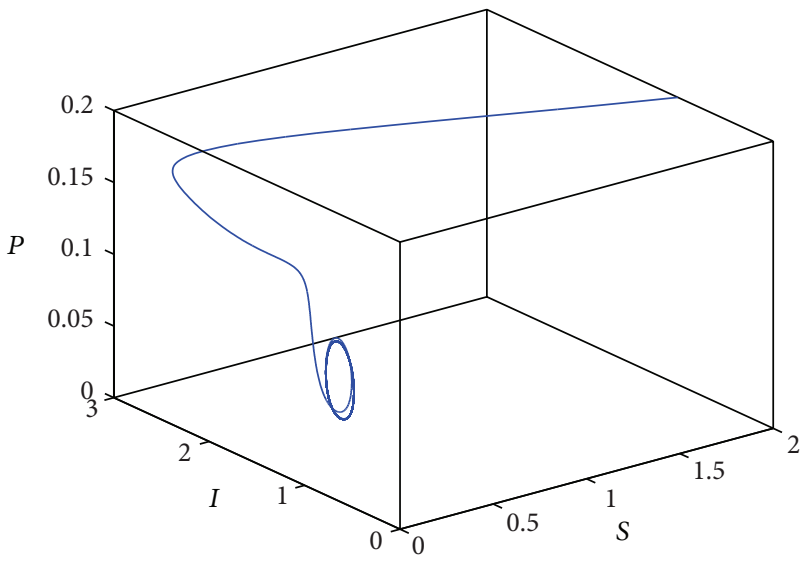

(b)

Figure 2: (a) The movement paths of $S, I$, and $P$ as functions of time $t$. The graph of the trajectory in $(S, I, P)$-Space is shown in $(\mathrm{b}) . R_{*}=$ $1.2288>1$. The disease is permanent.

\section{Numerical Simulation}

In this section, we present some numerical simulations to substantiate and augment our analytical findings of system (2) by means of the software Matlab.

In system (2), let $\Lambda(t)=0.6+0.3 \sin (2 t), d(t)=0.5+$ $0.2 \sin (2 t), e(t)=0.6+0.2 \sin (t), f(t)=0.05+0.04 \sin (t)$, $r(t)=0.5+0.4 \sin t, K(t)=0.1+0.08 \sin t, \alpha(t)=0.2+$ $0.01 \sin t, c_{1}(t)=0.18+0.1 \cos t, c_{2}(t)=0.5+0.1 \sin t, c_{3}(t)=$ $0.06+0.01 \sin t, c_{4}(t)=0.2+0.08 \cos t, g(t)=0.6+0.1 \sin t$, $\gamma=0.5$. Obviously, it is easy to verify that assumptions $(\mathrm{Cl})$ and (C2) hold. Let $\beta(t)=0.63+0.1 \sin t$; our results show that the upper threshold value $R^{*}=0.9898<1$, under which the conditions of Corollary 6 are satisfied. Hence, the disease will be extinct (see Figure 1). Increasing the infective rate to $\beta(t)=6+0.1 \sin t$, we can easily get the lower threshold value $R_{*}=1.2288>1$ and observe that all three species follow the system (2), which reaches a stable state. The conclusion of Corollary 5 is verified (see Figure 2). Also, we find that the force of infection or the contact rate is one of the important 


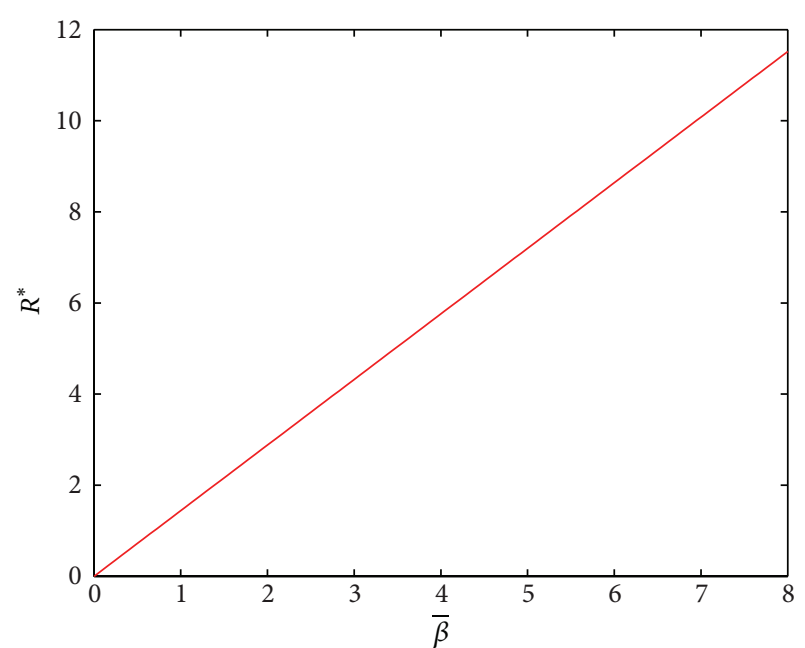

Figure 3: The graph of the upper threshold value $R^{*}$ versus $\bar{\beta}$.

parameters that control disease in an eco-epidemiological system. The graph for the relation of the basic reproduction ratio to $\bar{\beta}$ is obtained (see Figure 3 ).

Next, considering system (2) with initial conditions $(0.05,0.01,0.03),(0.6,0.1,0.1),(0.05,1.2,0.3),(0.6,0.8,0.6)$, and $(0.05,0.03,0.6)$, numerical simulation shows that the solution curves finally converge into a closed curve in threedimensional space, which imply that there exists a periodic solution of system (2) with the above conditions and it is globally attractive (see Figure 4). Therefore, we conjecture that if all the conditions of Corollary 5 hold, then system (2) has a positive periodic solution which is globally attractive.

Finally, it is well known that the disease plays an important role in a predator-prey system. Anderson and May [28] pointed that invasion of a resident predator-prey system by a new strain of parasites could cause destabilization and exhibits limit cycle oscillation. Thus to keep the system stability we have to make the system disease free. In the following, we will perform some numerical simulations to show the importance of recovery rate $f$ for controlling disease in an eco-epidemiological system. For system (2), we will discuss the effect of the mean value of recovery rate, $\bar{f}$, in the dynamics of the system. We choose $d(t)=0.5+0.2 \sin 2 t$, $c_{1}(t)=0.18+0.1 \cos t, \Lambda(t)=0.6+0.3 \sin 2 t, c_{2}(t)=$ $0.5+0.1 \sin t, e(t)=0.6+0.2 \sin t, r(t)=0.5+0.4 \sin t$, $K(t)=0.1+0.08 \sin t, \alpha(t)=0.2+0.01 \sin t, \beta(t)=$ $0.8+0.1 \sin t$, and the period $T=2 \pi$. As $\bar{f}$ varies in $[0,9]$, we obtain the graph for the relation of the basic reproduction ratio to $\bar{f}$ (see Figure 5 ). This figure shows that increasing the amplitude of periodically recovery rate reduces the risk of epidemic prevalence and the recovery rate on the system is also an important parameter for controlling disease in an eco-epidemiological system.

\section{Conflict of Interests}

The authors declare that they have no conflict of interests regarding the publication of this paper.

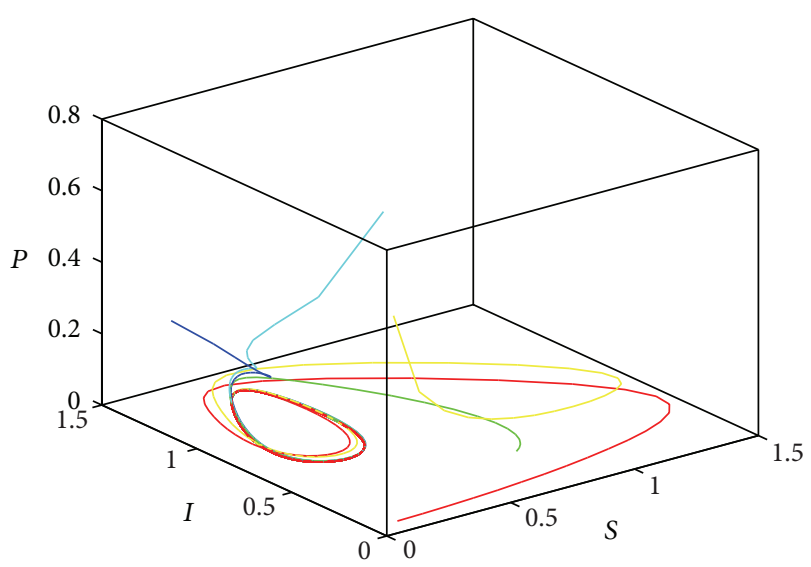

FIGURE 4: The existence of periodic solution of system $(2) . d(t)=$ $0.5+0.2 \sin (2 t), \Lambda(t)=0.6+0.5 \sin t, \beta(t)=3.2+\sin t, e(t)=$ $0.6+0.2 \sin t$, and $f(t)=0.05+0.045 \sin t$. The periodic solution is globally attractive.

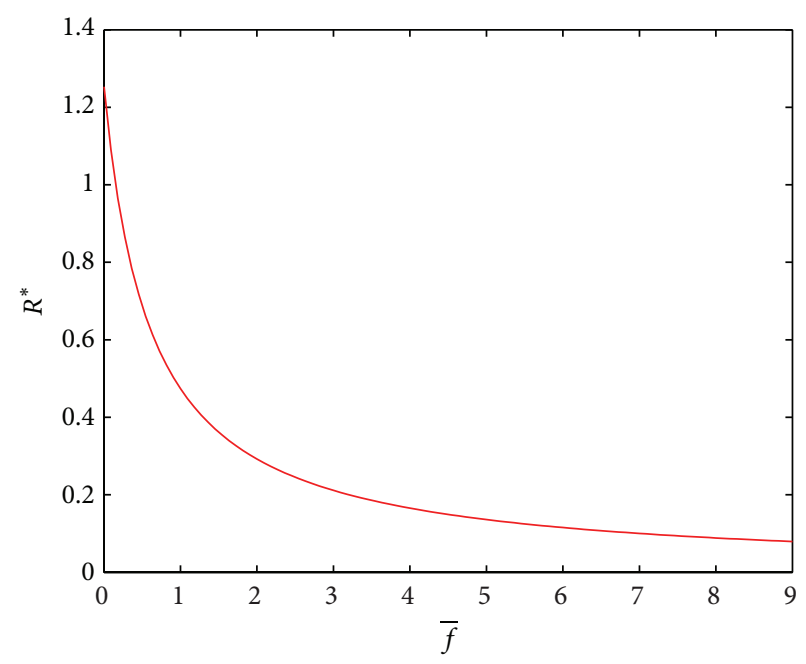

FIGURE 5: The graph of the upper threshold value $R^{*}$ versus $\bar{f}$.

\section{Acknowledgments}

The authors gratefully acknowledge the anonymous reviewers for their critical comments and suggestions on the original paper which have immensely improved the content and presentation of this version. The research has been supported by the Natural Science Foundation of China (11261004), the Natural Science Foundation of Jiangxi Province (20122BAB211010), the Foundation of Education Committee of Jiangxi (GJJ14441), and the Natural Science Foundation of Jiangxi University of Science and Technology (NSFJ2014G16).

\section{References}

[1] H. I. Freedman, Deterministic Mathematical Models in Population Ecology, vol. 57, Marcel Dekker, New York, NY, USA, 1980. 
[2] K. P. Hadeler and H. I. Freedman, "Predator-prey populations with parasitic infection," Journal of Mathematical Biology, vol. 27, no. 6, pp. 609-631, 1989.

[3] G. Pang, F. Wang, and L. Chen, "Extinction and permanence in delayed stage-structure predator-prey system with impulsive effects," Chaos, Solitons and Fractals, vol. 23, no. 2, pp. 631-643, 2009.

[4] Y. Chen, Z. Liu, and M. Haque, "Analysis of a Leslie-Gower-type prey-predator model with periodic impulsive perturbations," Communications in Nonlinear Science and Numerical Simulation, vol. 14, no. 8, pp. 3412-3423, 2009.

[5] S. Chatterjee and J. Chattopadhyay, "Role of migratory bird population in a simple eco-epidemiological model," Mathematical and Computer Modelling of Dynamcial Systems, vol. 13, no. 1, pp. 99-114, 2007.

[6] S. Chatterjee, "Alternative prey source coupled with prey recovery enhance stability between migratory prey and their predator in the presence of disease," Nonlinear Analysis: Real World Applications, vol. 11, no. 5, pp. 4415-4430, 2010.

[7] K. I. Kim, Z. Lin, and L. Zhang, "Avian-human influenza epidemic model with diffusion," Nonlinear Analysis. Real World Applications, vol. 11, no. 1, pp. 313-322, 2010.

[8] S. Chatterjee, S. Pal, and J. Chattopadhyay, "Role of migratory birds under environmental fluctuation - a mathematical study," The Journal of Biological Systems, vol. 16, no. 1, pp. 81-106, 2008.

[9] J. H. Rappole, S. R. Derrickson, and Z. Hubálek, "Migratory birds and spread of West Nile virus in the Western Hemisphere," Emerging Infectious Diseases, vol. 6, no. 4, pp. 319-328, 2000.

[10] D. J. D. Earn, J. Dushoff, and S. A. Levin, "Ecology and evolution of the flu," Trends in Ecology and Evolution, vol. 17, no. 7, pp. 334340, 2002.

[11] Y. Zhang, S. Gao, and Y. Liu, "Analysis of a nonautonomous model for migratory birds with saturation incidence rate," Communications in Nonlinear Science and Numerical Simulation, vol. 17, no. 4, pp. 1659-1672, 2012.

[12] H. Qiu, M. Liu, K. Wang, and Y. Wang, "Dynamics of a stochastic predator-prey system with Beddington-DeAngelis functional response," Applied Mathematics and Computation, vol. 219, no. 4, pp. 2303-2312, 2012.

[13] X.-Q. Liu, S.-M. Zhong, B.-D. Tian, and F.-X. Zheng, "Asymptotic properties of a stochastic predator-prey model with Crowley-Martin functional response," Journal of Applied Mathematics and Computing, vol. 43, no. 1-2, pp. 479-490, 2013.

[14] P. Crowley and E. Martin, "Functional response and interference within and between year classes of a dragonfly population," Journal of the North American Benthological Society, vol. 8, no. 3, pp. 211-221, 1989.

[15] J. Beddington, "Mutual interference between parasites or predators and its effect on searching efficiency," Journal of Animal Ecology, vol. 44, pp. 331-341, 1975.

[16] D. DeAngelis, R. Goldsten, and R. Neill, "A model for trophic interaction," Ecology, vol. 56, pp. 881-892, 1975.

[17] M. P. Hassell and G. C. Varley, "New inductive population model for insect parasites and its bearing on biological control," Nature, vol. 223, no. 5211, pp. 1133-1137, 1969.

[18] K. Wang and Y. L. Zhu, "Permanence and global asymptotic stability of a delayed predator-prey model with Hassell-Varley type functional response," Bulletin of the Iranian Mathematical Society, vol. 37, no. 3, pp. 197-215, 2011.

[19] S. Hsu, T. Hwang, and Y. Kuang, "Global dynamics of a predator-prey model with Hassell-Varley type functional response,"
Discrete and Continuous Dynamical Systems B: A Journal Bridging Mathematics and Sciences, vol. 10, no. 4, pp. 857-871, 2008.

[20] S. Pathak, A. Maiti, and G. P. Samanta, "Rich dynamics of a food chain model with Hassell-Varley type functional responses," Applied Mathematics and Computation, vol. 208, no. 2, pp. 303317,2009 .

[21] H. K. Kim and H. Baek, "The dynamical complexity of a predator-prey system with Hassell-Varley functional response and impulsive effect," Mathematics and Computers in Simulation, vol. 94, pp. 1-14, 2013.

[22] K. Wang, "Periodic solutions to a delayed predator-prey model with Hassell-Varley type functional response," Nonlinear Analysis. Real World Applications, vol. 12, no. 1, pp. 137-145, 2011.

[23] M. J. Wonham, T. de-Camino-Beck, and M. A. Lewis, "An epidemiological model for West Nile virus: invasion analysis and control applications," Proceedings of the Royal Society B: Biological Sciences, vol. 271, no. 1538, pp. 501-507, 2004.

[24] E. Venturino, "The effects of diseases on competing species," Mathematical Biosciences, vol. 174, no. 2, pp. 111-131, 2001.

[25] T. Zhang and Z. Teng, "On a nonautonomous SEIRS model in epidemiology," Bulletin of Mathematical Biology, vol. 69, no. 8, pp. 2537-2559, 2007.

[26] Z. Teng and Z. Li, "Permanence and asymptotic behavior of the $N$-species nonautonomous Lotka-Volterra competitive systems," Computers \& Mathematics with Applications, vol. 39, no. 7-8, pp. 107-116, 2000.

[27] X. Feng, Z. Teng, and L. Zhang, "Permanence for nonautonomous $n$-species Lotka-Volterra competitive systems with feedback controls," The Rocky Mountain Journal of Mathematics, vol. 38, no. 5, pp. 1355-1376, 2008.

[28] R. M. Anderson and R. M. May, "The invasion, persistence and spread of infectious diseases within animal and plant communities," Philosophical transactions of the Royal Society of London B, vol. 314, no. 1167, pp. 533-570, 1986. 


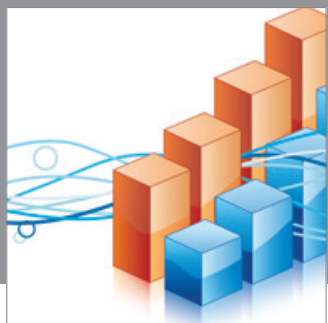

Advances in

Operations Research

mansans

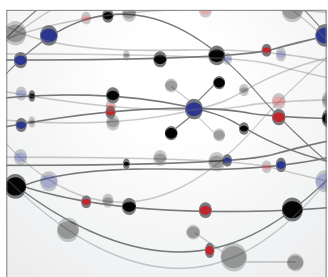

The Scientific World Journal
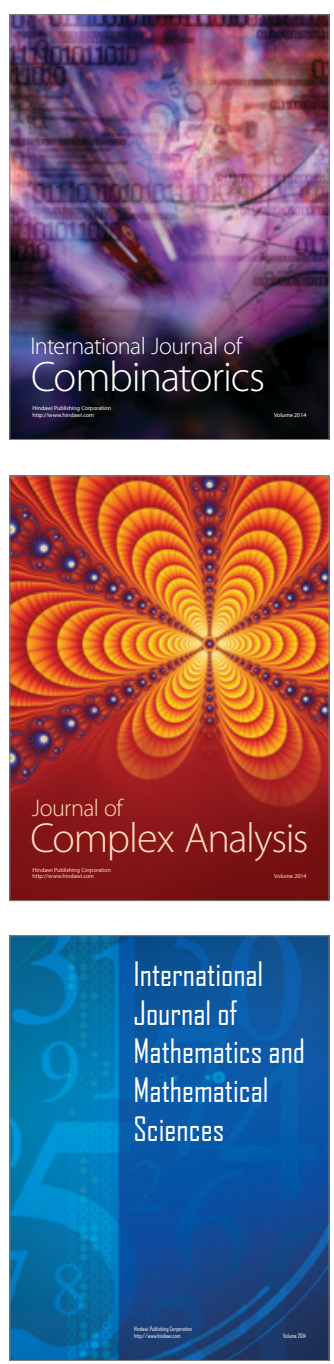
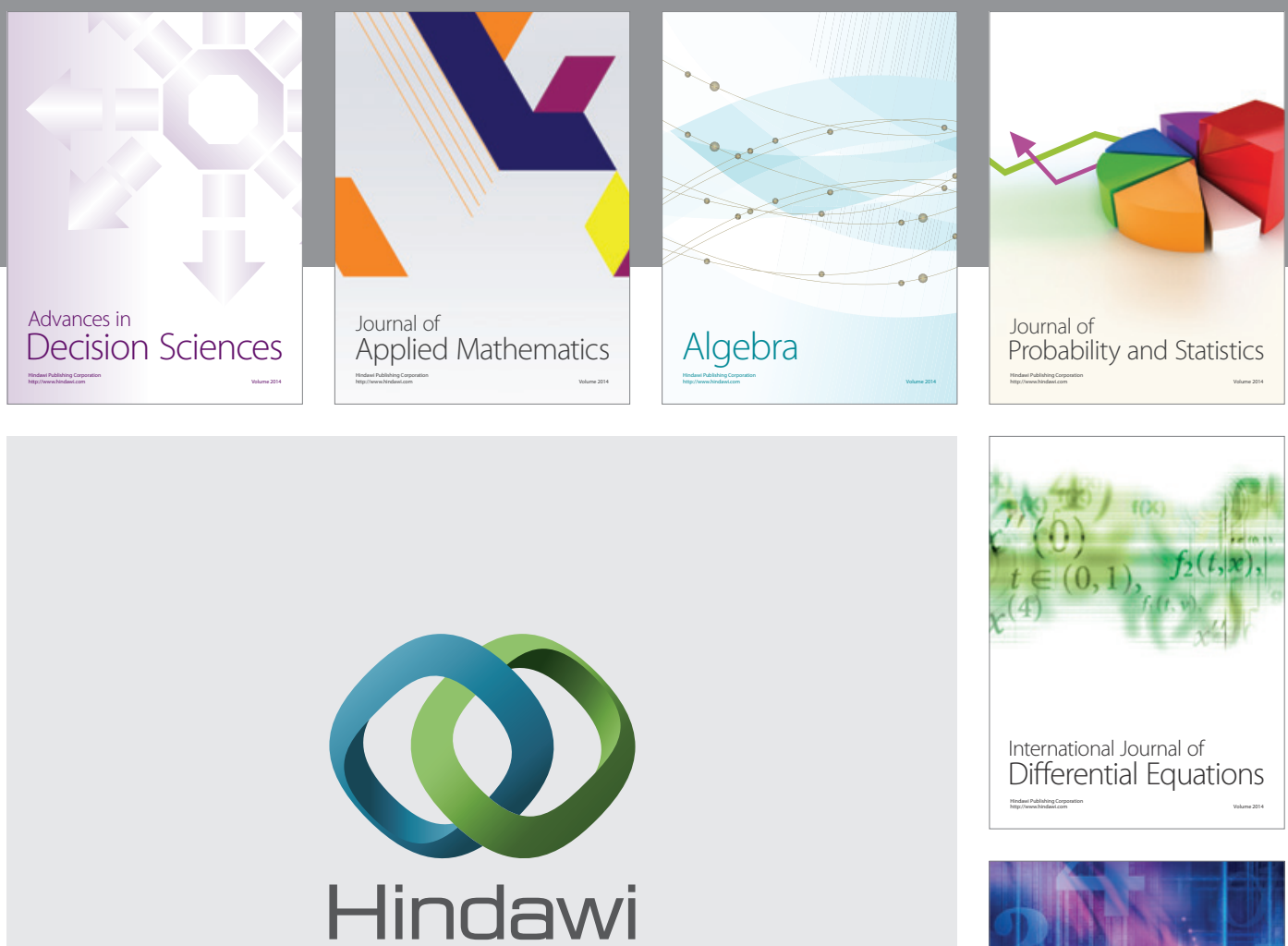

Submit your manuscripts at http://www.hindawi.com
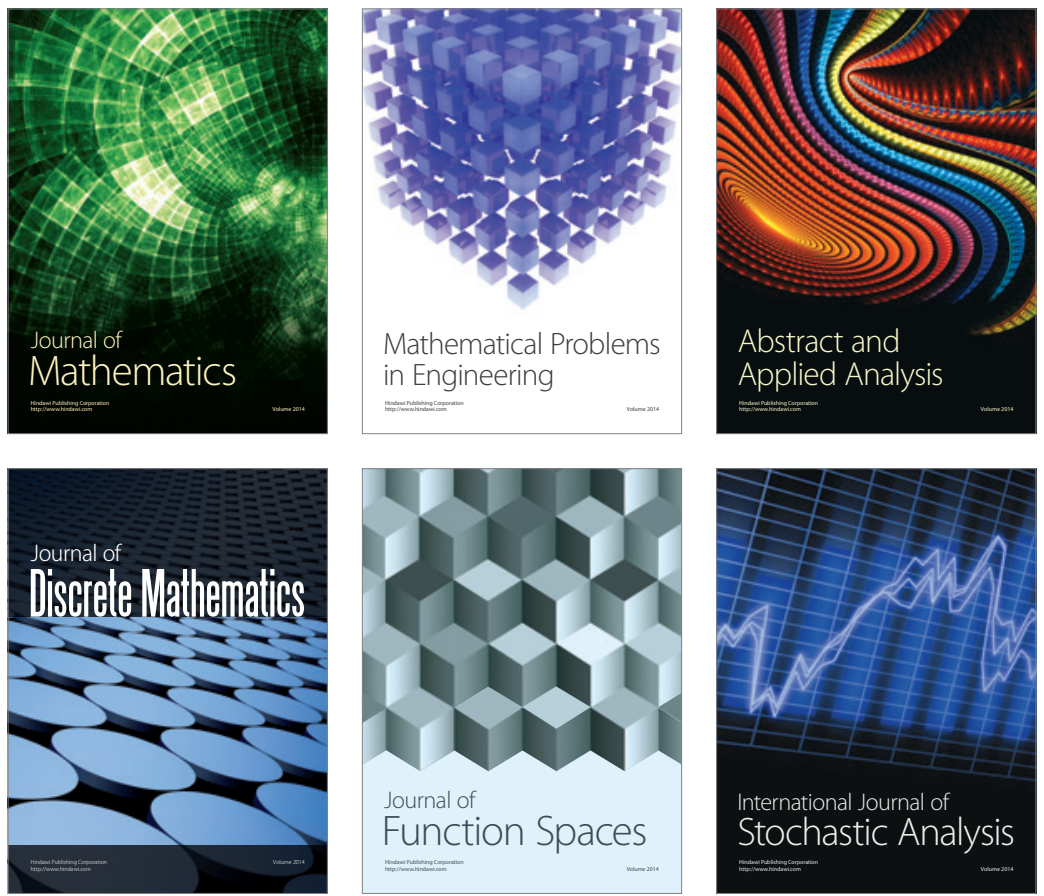

Journal of

Function Spaces

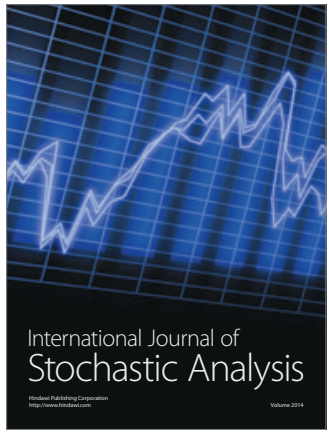

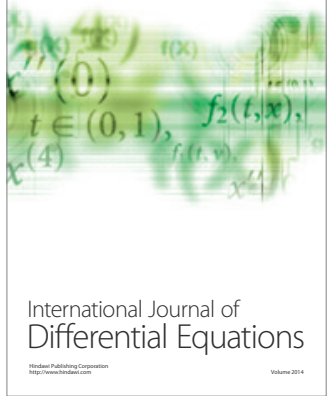
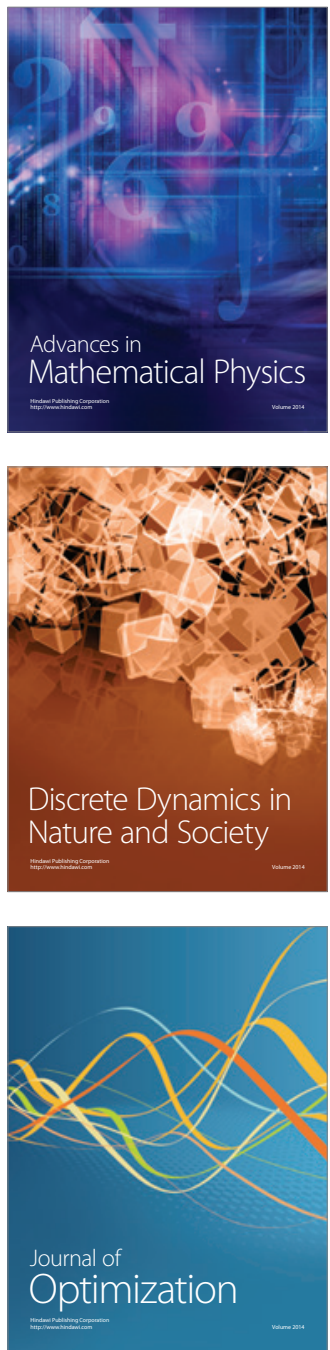